\title{
A Spline-Merging -Pattern Model of Toll Plaza
}

\author{
Feiliang $\mathrm{Hu}$ \\ Department of Electronic and Communication Engineering, North China Electric Power University, \\ Baoding 071003, China \\ www.734844143@qq.com
}

\begin{abstract}
In working out a model that would make optimization of the feature of toll plaza, such as shape ,size and merging-pattern, our team decided to apply the Queuing Theory and simulation software Vissim to figure out parameters of microcosmic traffic model .Studying on a large sum of relevant data. We then create a fitting function to describe the shape of toll plaza called splinemerging-pattern model to optimize merging based on common design and standard for design. By simulating the ability of our model suitable for different combinations of $\mathrm{L} / \mathrm{B}$, the proportion of three types of tollbooths.
\end{abstract}

Keywords: Queuing Theory, multi-merging-pattern.

\section{Introduction}

When exiting the tollbooths in a barrier toll, vehicles must "fan in" from the larger number of tollbooth egress lanes to the smaller number of regular travel lanes. A toll plaza is the area of the highway needed to facilitate the barrier toll, consisting of the fan-out area before the barrier toll, the toll barrier itself, and the fan-in area after the toll barrier.

Three types of tollbooths such as conventional tollbooths, exact-change tollbooths, and electronic toll collection booths.

\section{Solution}

We discuss the queuing model before tollbooths and merging pattern after toll. What's more, we need establish model and select different parameters to evaluate the performance of our models.

\section{Single channel service[1]}

When using $\mathrm{M} / \mathrm{M} / 1$, we set the vehicle average arrival rate as $\lambda$ (cars/s), the system average service rate as $\mu($ cars $/ s)$, the traffic intensity as $\rho$.

$\rho=\lambda / \mu<1$

By the recurrence formula, the probability of $\mathrm{n}$ car in the system

By the probability properties

$$
\begin{aligned}
& \sum_{n=}^{\infty} P= \\
& P_{0}=1-\rho \\
& P_{n}=(1-\rho) \rho^{n}
\end{aligned}
$$

The queue length Len--according to the system, expecting vehicle number, we set the probability of $\mathrm{n}$ cars as Pn, the length is 
The expected time $\mathrm{W}$ of Vehicle staying in the system in toll station queuing system is a random variable, we can prove that it obey the parameter of $\mu-\lambda$ Negative exponential distribution, and the distribution function and density function is

$$
\begin{aligned}
& F(w)=1-e^{-(\mu-\lambda) w} \\
& f(w)=(\mu-\lambda) e^{-(\mu-\lambda) w}
\end{aligned}
$$

The expected waiting time Wq of Vehicles in the system should minus the average service time, namely

$$
W_{q}=W-\frac{1}{\mu}=\frac{1}{\mu-\lambda}-\frac{1}{\mu}=\frac{\mu}{\mu(\mu-\lambda)}=\frac{L_{q}}{\lambda}
$$

\section{Referring to[2], we design such common toll plaza(at Fig 1).}

\section{Toll Lane Width}

The distance between kerb faces is between 3.0 and 3.3 meters within the toll lanes of major existing UK toll plazas. Toll lane widths within this range help to slow traffic and guide the driver closer to the toll collector or ACM bucket. A greater width should be considered at the height of HGV.

The toll island width at existing facilities varies between $1.2 \mathrm{~m}$ and $3.0 \mathrm{~m}$, although most islands are 1.8 to 2.1 metres wide. The overall plaza width is calculated after establishing the number of lanes required, the island widths and the toll lane widths.

The length of the Queue Zone should be sufficient to accommodate the design flow. A traffic simulation exercise should be undertaken to assess the extent of queues at the maximum annual peak hour flow to ensure that they do not extend back to the approach carriageway. On exiting the toll lane, traffic enters the Recovery Zone where vehicles accelerate to regain speed. Beyond the Recovery Zone is the Departure Zone which reduces in width to funnel the traffic back down to the departure carriageway cross section."

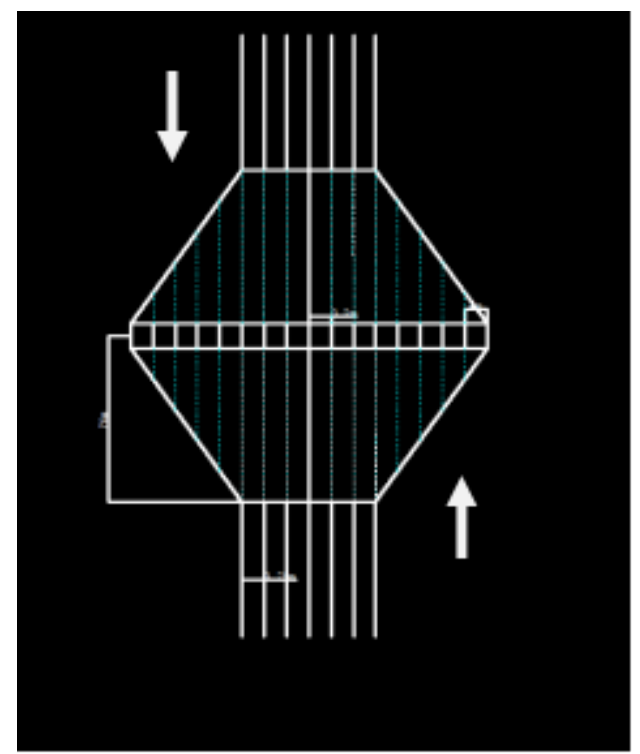

Fig.1 Common toll plaza

Solving function by matlab and achieving the area of toll plaza by AutoCAD:

In light traffic, get figure 2 ( $\mathrm{f}(\mathrm{x}) /$ matrix of three types) 


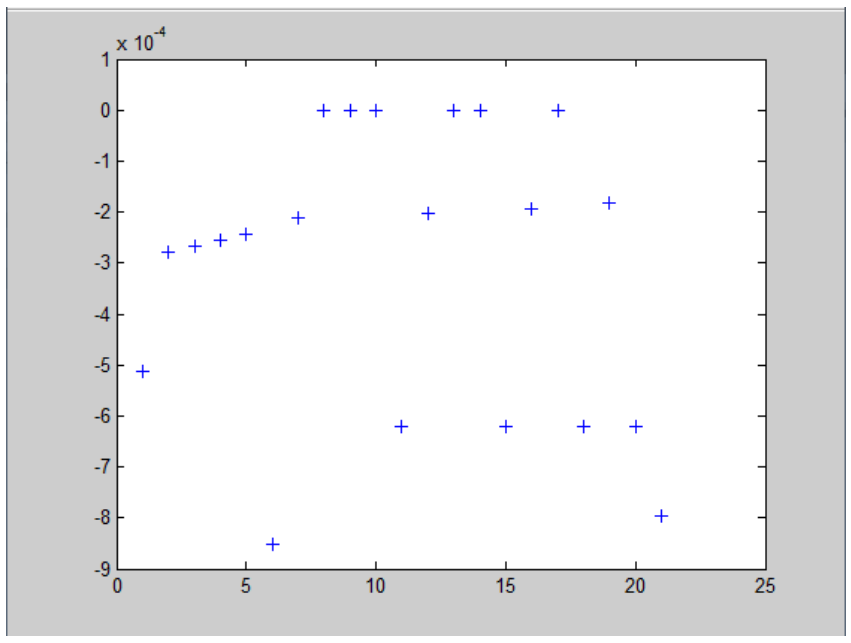

Fig 2 Matrix of three types

Other than those point $(f(x)=0)$, when Min $f(x)$ we get $x=\left[\begin{array}{lll}5 & 1 & 2\end{array}\right]$.

Choosing five human-staffed tollbooths, tone exact-change(automated) tollbooth and wo electronic toll collection booth, $\mathrm{S}=1697.4 \mathrm{~m}^{2}$

\section{Simulate and Optimize on vehicle throughput(light/heavy)}

We use Poisson distribution of traffic flow (the formula below)to calculate probability of each lane in 20s (at Fig3)

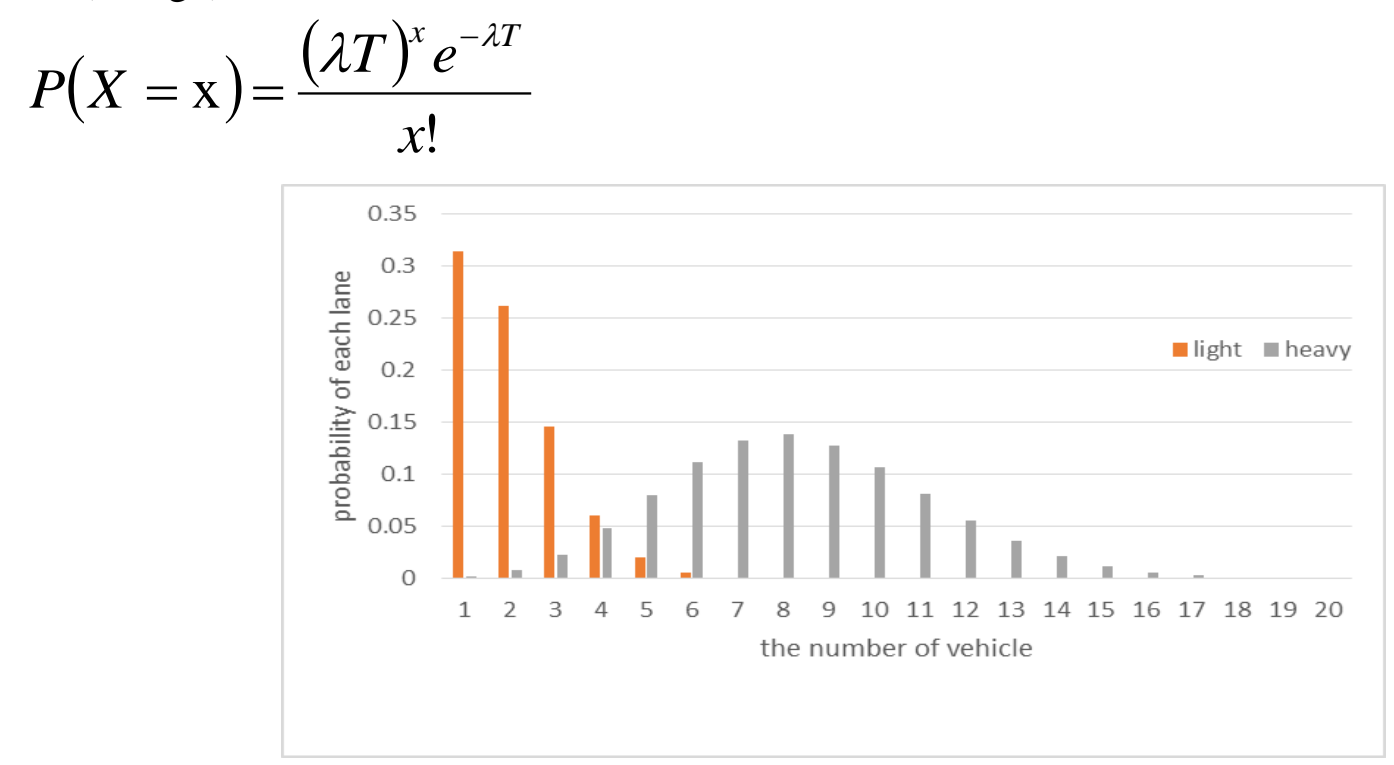

Fig.3 Distribution of traffic flow

We can find that the distribution of probability of each lane in 20s in the different types of vehicle throughput are obviously. So we need simulate and optimize our model.

The accident prevention

We can add the number of tollbooths on each lane in case of traffic congestion, and adopt adjacent open emergency toll channel, traffic flow guidance and traffic lights limiting speed.

Regular peak hour delays could be addressed by introducing higher toll charges during peak periods with the aim of spreading the peak demand over a longer period" [3]

The figure below (Fig4)is the change of capacity with the vph, when appear congestion, the capacity decreases sharply. 


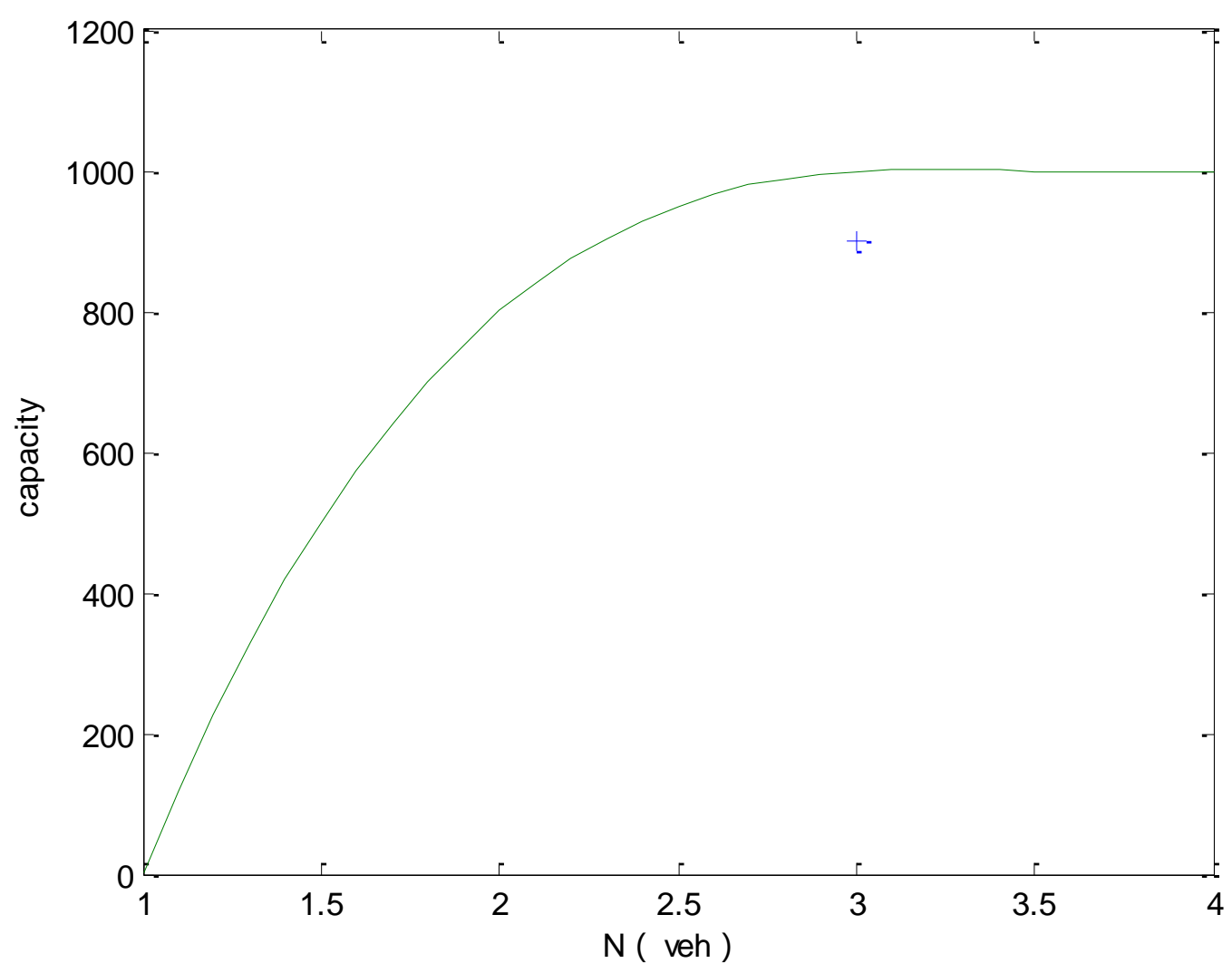

Fig.4 Capacity

\section{Summary}

According to relevant study, it is urgent to change the existing model of toll plaza and make different plans for different toll lanes. What's more, reducing congestion is beneficial to environment and health.

\section{References}

[1] Weiwei Liu, Weisi Ma. Analysis of queuing theory model in the highway toll station

[2] Information on www.standardsforhighways.co.uk_ha_standards_dmrb_vol6_section3_ta9808

[3] Information on www.standardsforhighways.co.uk_ha_standards_dmrb_vol6_section3_ta9808 